\title{
Different Kinds of Chaotic Oscillations in the Belousov-Zhabotinskii Reaction
}

\author{
Klaus Wegmann \\ Institut für Chemische Pflanzenphysiologie der Universität Tübingen \\ and \\ Otto E. Rössler \\ Institut für Physikalische und Theoretische Chemie der Universität Tübingen and \\ Institut für Theoretische Physik der Universität Stuttgart
}

Z. Naturforsch. 33 a, 1179-1183 (1978); received July 19, 1978

Following earlier evidence for chaotic time behaviour in the open flow continuous stirred Belousov-Zhabotinskii reaction based on a single measured observable, now two-dimensional phase plots are presented. The potential of a bromide ion sensitive electrode was plotted against the electrochemical potential. In the two-dimensional observation space obtained, both apparent limit cycle oscillations and apparent chaotic oscillations were observed. Of the latter, type 1 resembles a double-limit cycle system with fluctuation-triggered transitions between the two regimes (exogenous chaos); type 2 is analogous, but here the irregular transition between the two oscillatory regimes gives the impression of being, in principle, independent of exogenous noise. If so, this flow may be related to one of the chaotic flows with saddle point known (for example, the Lorenz attractor). Type 3, only observed as a transient so far, looks like a two-dimensional projection of screw type chaos, which is one of the simplest types of deterministic chaos. Type 4 corresponds either to a fluctuation-triggered monoflop (exogenous chaos) or to folded-strip (spiral) type chaos.

\section{Introduction}

Nonlinear deterministic continuous oscillators of more than two variables are in many cases capable of more complicated types of oscillations beyond the ordinary limit cycle. The most prominent class of complicated oscillations became known recently under the name of "chaos" [1] (cf. [2]). Chaotic oscillations are characterized [1] (a) by the presence of an infinite number of (mostly unstable) periodic solutions plus an uncountable number of nonperiodically oscillating solutions and (b) by the phenomenon of "hyperbolicity", meaning that closely adjacent initial conditions lead to rapidly diverging trajectories for most initial conditions. An example is Lorenz's "butterfly effect" which states that even if the equations and initial conditions determining the weather were known with absolute accuracy, so that the future time course could be computed in principle, a single wingclapping by a butterfly would suffice to render the future time course completely different [3]. The oldest example of chaos is the well-known "analytically insoluble" restricted three-body problem of celestial mechanics (see [4] for a recent account).

Reprint requests to Prof. O. E. Rössler, Division of Theoretical Chemistry, University of Tübingen, Auf der Morgenstelle 8, D-7400 Tübingen, Fed. Rep. of Germany.
The simplest everyday example of a chaotic oscillation is the irregularly dripping faucet [5].

A first three-variable abstract reaction system capable of chaotic oscillations was proposed in [6]. This system at the same time can be considered as a "blown-up" version of an earlier described 2-variable qualitative model of the Zhabotinskii reaction [7]. For a somewhat more complicated alternative blowing up of the same equation, see [8]. Both models yield spiral type chaos [9].

Like the simplest spiral-type chaos producing equation [10,2], the simplified models of the Zhabotinskii reaction mentioned probably can produce screw-type chaos too. More complicated - and more realistic - models of the Zhabotinskii reaction may exhibit additional (known and unknown) types of chaos. A seven-variable model with complicated oscillations (although not chaos so far) has been simulated recently [11].

The rate equations of the Belousov-Zhabotinskii reaction are known in great detail [12], although the range of a few parameters has yet to be determined. In spite of this almost complete chemical eludication, the qualitative dynamics implicit in the equation are still largely unknown. This is not astonishing, however, since establishing a complete catalog of the possible types of qualitative behavior of a given nonlinear system (called the "logos" of 
that system [13]) is exceedingly difficult. Moreover, the proneness of most nonlinear systems to exhibiting chaotic oscillations was virtually unsuspected until recently, although, according to Lefschetz [14], Poincaré (who discovered the phenomenon) knew about its prevalence. In reaction kinetics, the earliest prediction concerned "strange attractors" [15] (on the basis of four coupled oscillators; for two simpler cases, see [16]. So it is not astonishing that the first evidence of chaos (or at least a complicated limit cycle) in the Zhabotinskii reaction, reported under the name of a "double frequency mode" of oscillation [17], went unnoticed.

Chaos in the Zhabotinskii reaction was first explicitly described by Schmitz et al. [18]. The authors proposed applicability of the principle of spiral-type chaos [6] for explaining certain complicated $x-t$ plots which they had been unable to interpret before due to lack of a theoretical paradigm [19].

Unaware of these experimental observations, we attempted to verify the prediction made in [6] that a chaotic mode should be easy to find in the Zhabotinskii reaction. A first irregular time course found was tentatively interpreted as an example of screw-type chaos $[20,21]$ and the necessity of further experimental work involving more than a single measured variable was stressed [21].

In the following, we present a set of two-dimensional plots (first presented in [22]) which allow us to address in somewhat greater detail the two main questions that are still open: (1) Is "potentially endogenous" chaos possible in the Zhabotinskii reaction? (2) If so, to which type or types does it belong?

\section{Materials and Methods}

All chemicals used were analytical grade obtained from Merck, Darmstadt. The concentration of

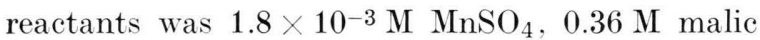
acid, $0.015 \mathrm{M} \mathrm{KBrO}_{3}$, and $1.83 \mathrm{M} \mathrm{H}_{2} \mathrm{SO}_{4}$ [23]. The reaction was started by rapid mixing of a solution I (containing per liter $0.34 \mathrm{~g} \mathrm{MnSO}_{4} \cdot \mathrm{H}_{2} \mathrm{O}, 54 \mathrm{~g}$ malic acid, and $200 \mathrm{~g} \mathrm{H}_{2} \mathrm{SO}_{4}$ ) and a solution II (23.3 g $\mathrm{KBrO}_{3}$ per liter distilled $\mathrm{H}_{2} \mathrm{O}$ ) in a ratio of $50: 6$. The two solutions were then continuously injected with a peristaltic pump (Pharmacia P-3) at that same ratio. The flow rates are indicated in the figure legends. The volume of the reaction mixture was $36 \mathrm{ml}$ and it was kept constant by an overflow mechanism. During the whole experiment the reaction mixture was agitated by a magnetic stirrer. The temperature was kept constant $\left( \pm 0.05^{\circ} \mathrm{C}\right)$ at the values given in the figures. The electrochemical potential was determined with a platinum electrode. A bromide ion selective solid state electrode (Br-125, TOA Electronics Ltd., Tokyo) was used for the second observable. An $\mathrm{Ag} / \mathrm{AgCl}$ electrode was used as common reference for both potentials. An HP 7005 B X-Y analog recorder was used. The voltage amplification factor was the same in all figures (see Fig. 5 for calibration bars).

\section{Results}

In Fig. 1, an oscillation of limit cycle type is presented. The picture apparently corresponds to a single-looped limit cycle in $n$ space, projected onto a plane. The upper right-hand corner seems to correspond to a tilted portion (side view). There is a certain amount of "noise" in the picture.

The picture shown in Fig. 2 looks similar for the greater part of the oscillatory cycle. However, the trajectory went along a different, apparently also well defined path two times during the observation interval, whereby the incident could not predicted. The picture thus seems to imply that there is a separatrix between two stable paths (a pseudoseparatrix in the sense of FitzHugh [24] would also

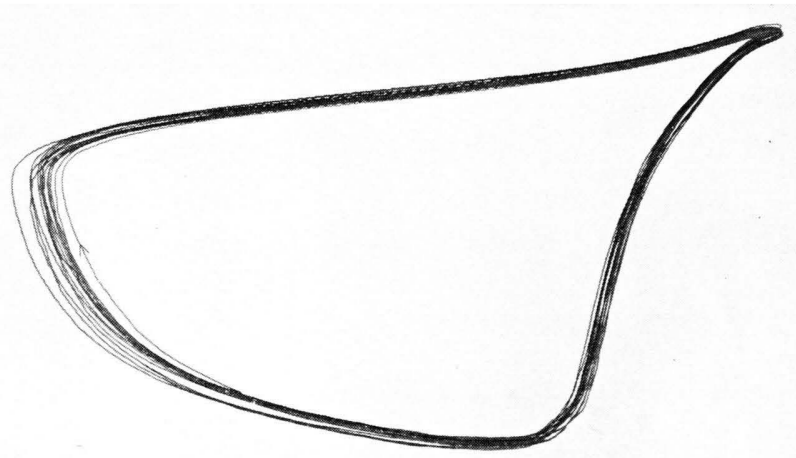

Fig. 1. "Limit cycle". Two-dimensional plot of electrochemical potential (abscissa) vs. potential of a bromide ion sensitive electrode (ordinate) in the well-stirred BelousovZhabotinskii reaction under isothermal open flow conditions. Scaling as in Figure 5. Flow rate: $2.7 \mathrm{ml} / \mathrm{min}$, temperature: $30^{\circ} \mathrm{C}$. Bromate concentration: $0.045 \mathrm{M} / 1$. Other values as described in Section 2. The system had been run at the same flow rate for two hours before the measurement was taken. 


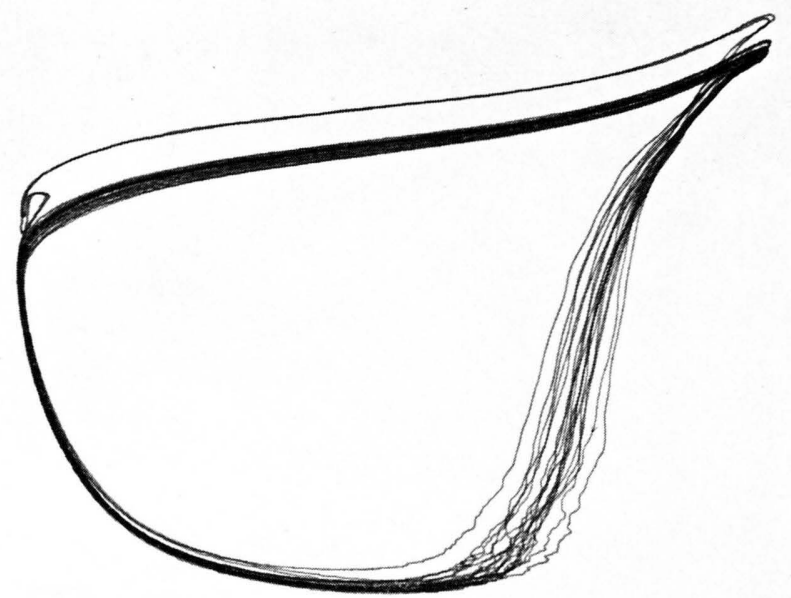

Fig. 2. "Double limit cycle". Scaling as in Fig. 5. Parameters as in Section 2 . Flow rate: $1.9 \mathrm{ml} / \mathrm{min}$, temperature: $24^{\circ} \mathrm{C}$. The system had been run at a slightly lower flow rate until one hour before this plot was made (stationary regime) [29].

be sufficient). If the separatrix comes close enough to the main path (limit cycle) at one point during the oscillatory cycle, exogenous fluctuations suffice to throw the system's representative state over into the adjacent basin (or quasi-basin) from time to time. The result is a kind of "pseudo-chaos", since the corresponding deterministic system would not show it.

Figure 3 shows a related regime. This time, however, a "continuous curtain" of transitions is

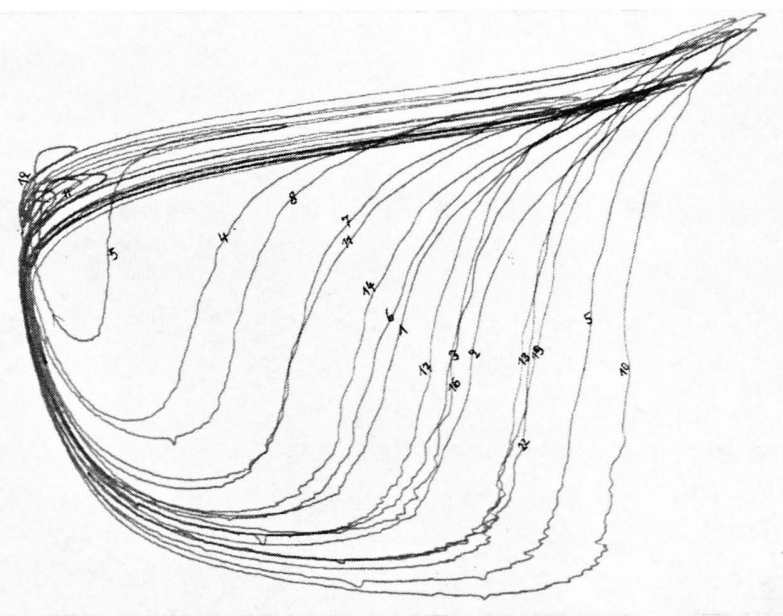

Fig. 3. "Endogenous chaos". Conditions as in Figure 2. Flow rate: $1.7 \mathrm{ml} / \mathrm{min}$, temperature: $22.8^{\circ} \mathrm{C}$. Stationary regime. observed in the right-hand portion of the picture. At the same time, the formation of a multiply folded "screw-like" transition is evident in the lefthand part of the picture. Even though exogenous fluctuations are still present, a picture like this can probably also be generated "endogenously", that is, deterministically. The flow seems to take place on a folded 2-dimensional manifold in state space. Nonetheless, this suggested picture is still rather complicated when compared with that of a simple example of deterministic chaos (see Fig. 12 in Ref. [2]). A flow like that of Fig. 3, but produced deterministically, would have to be classified as an example of "composite" chaos (see Ref. [2], Fig. 14 for several 3-variable examples).

Figure 4 shows a measured curve that looks fairly similar to the ideal case of screw-type chaos (cf. Fig. 13 in Ref. [9]). Unfortunately, this regime was observed as a transient only: it occurred 20 minutes after changing the flow rate from 2.0 to $6.7 \mathrm{ml} / \mathrm{min}$ and lasted 10 minutes. It may nonetheless correspond to a regime that can be evoked at a fixed set of parameters also: Following so relatively long a time (20 minutes) after a change of flow rates, the remaining automatic readjustment of parameters has become rather slow already. Therefore, there is a chance that a stationary configuration of parameters yielding the same behaviour may be found.

Figure 5 finally shows an irregular motion that is not easily interpretable. The picture may correspond to a monoflop (excitable) state of the system. (Excitable behaviour in the Zhabotinskii reaction was first observed by Winfree [25].) The motion was indeed somewhat slower in the upper righthand corner so that presence of an excitable stable

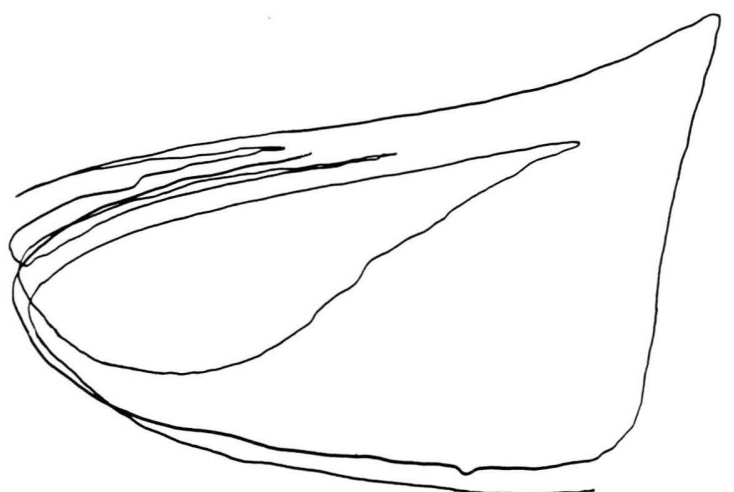

Fig. 4. "Screw type chaos". (Transient). Conditions as in Fig. 2. Flow rate: see text. Temperature: $22.3^{\circ} \mathrm{C}$. 


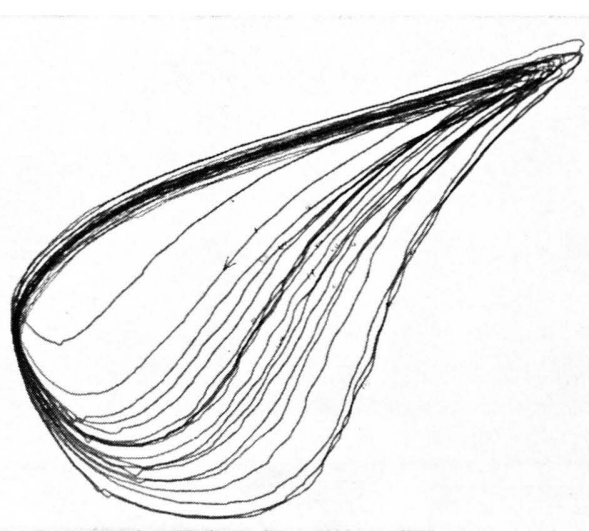

Fig. 5. "Exogenous chaos" of monoflop type (or, alternatively, endogenous folded-strip type chaos). Conditions as in Figure 2. Flow rate: $2.2 \mathrm{ml} / \mathrm{min}$. Temperature: $22.8^{\circ} \mathrm{C}$. Stationary regime. Scaling: Both calibration bars have a length of $10 \mathrm{mV}$. The right-hand one is located at about $-150 \mathrm{mV}$, the left-hand one around $500 \mathrm{mV}$. More positive values point downward or to the right, respectively.

steady state in this region cannot be excluded; in this case the observed varying amplitudes of excursion would be caused by the varying size of the triggering fluctuations. However, the smoothness of the curves in the right-hand corner speaks against such an interpretation, since excitable steady states usually look different, more "scrambled", in these plots [26].

An alternative explanation therefore postulates that an oscillation takes place on a smooth 2-dimensional submanifold or strip in state space, the righthand corner corresponding to a lateral view of the strip. The varying size of the excursions could then be explained by the presence of a lateral "folding over" of the strip, such that a combination of a Möbius strip with an ordinary strip results [6]. Such folded-strip type chaos has indeed been observed in 3 -variable deterministic equations [2]. It is topologically equivalent to spiral type chaos [6].

The last proposed explanation of Fig. 5 is supported by the fact that successive excursions statistically tend to be of opposite amplitudes. If this interpretation is right, the role of exogenous noise mainly consists in blurring the next amplitude law (successive amplitudes being a non-monotone function of the last amplitude) that is characteristic of spiral type chaos [6]. As Fig. 6 shows, successively recorded "amplitudes" (obtained from Fig. 5 as successive maximum deviations in a "southwesterly" direction), when they are plotted each as

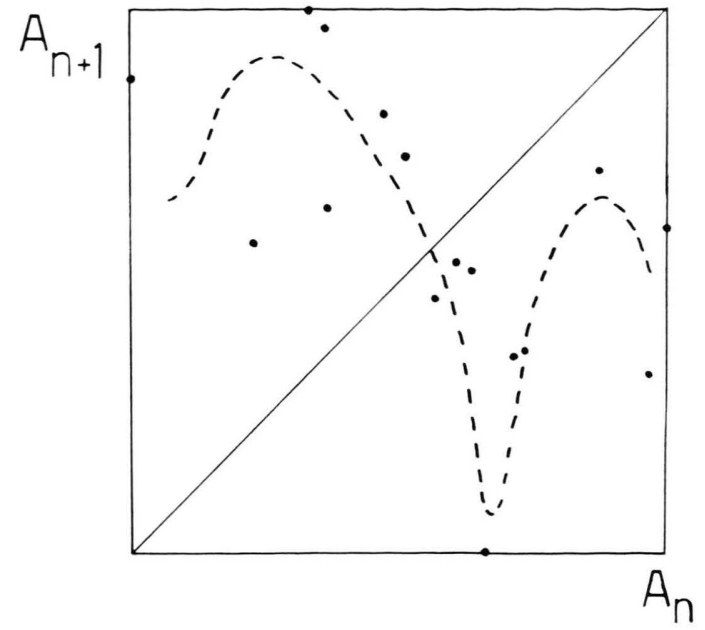

Fig. 6. "Next-amplitude map" for the flow of Fig. 5 (see text). The dashed line is not a calculated curve.

a function of the last, may give the impression of an underlying non-monotonic curve that is masked by external noise. The present Figure, however, is much less convincing than an analogous curve that was obtained earlier for a different chemical oscillator, viz. the horseradish peroxidase (Yamazaki) oscillator, on the basis of a single measured time curve [27].

\section{Conclusion}

To prove that "endogenous" chaos is possible in the Zhabotinskii reaction, at least three appropriate observables are required. In addition, a low noise level is desirable. If then one of the typical chaosproducing two-dimensional submanifolds in state space [2] were detectable (although necessarily blurred), the question whether the observed "chaotic" regime would remain the same in the absence of external noise would become academic. That is to say, the existence of chaos can, in principle, be made probable by experiment to a degree which comes close to an empirical proof. However, only certain simple types of chaos can be empirically identified easily in this way. Diagnostic criteria for the presence of endogenous chaos can therefore only be of the "sufficient" type.

The preliminary results obtained so far seem to justify further experimentation. They also call for more detailed numerical investigations of the available rate equations and simplified models of the Zhabotinskii reaction [28].

We thank Art Winfree for discussions. 
[1] T. Y. Li and J. A. Yorke, Amer. Math. Mth. 82, 985 (1975).

[2] O. E. Rössler, Continuous Chaos. In: Synergetics, A Workshop (H. Haken, ed.), pp. 184-197. SpringerVerlag, Berlin 1977.

[3] E. N. Lorenz, J. Atmos. Sci. 20, 130 (1963).

[4] J. Moser, Stable and Random Motions in Dynamical Systems with Special Emphasis on Celestial Mechanics. Princeton University Press, Princeton 1973.

[5] O. E. Rössler, Chemical Turbulence. - A Synopsis. In: Synergetics, A Workshop (H. Haken, ed.), pp. 174-184. Springer-Verlag, Berlin 1977.

[6] O. E. Rössler, Z. Naturforsch. 31 a, 259-264 (1976).

[7] O. E. Rössler, J. Theor. Biol. 36, 413-417 (1972).

[8] J. Tyson, preprint, J. Math. Biol. (1978).

[9] O. E. Rössler, J. Math. Biol. 39, 275-289 (1977).

[10] O. E. Rössler, Phys. Letters 57 A, $397-398$ (1976).

[11] K. Showalter, R. M. Noyes, and K. Bar-Eli, A Modified Oregonator Model Exhibiting Complicated Limit Cycle Behavior in a Flow System. Preprint 1978.

[12] J. J. Field, E. Körös, and R. M. Noyes, J. Amer. Chem. Soc. 8649-8664 (1972).

[13] R. Abraham, Vibrations and the Realization of Form. In: Evolution and Consciousness (E. Jantsch and C. H. Wattington, eds.), pp. 134-149. AddisonWesley, London, Tokyo 1976.

[14] S. Lefschetz, Geometric Differential Equations : Recent Past and Proximate Future. In: Differential Equations and Dynamical Systems (J.H. Hale and J.P. LaSalle, eds.), pp. 1-14. Academic Press, New York 1976.

[15] D. Ruelle and F. Takens, Commun. Math. Phys. 20, 167 (1971).

[16] O. E. Rössler and P. J. Ortoleva, Strange Attractors in Three-Variable Reaction Systems, Springer Lecture Notes in Biomathematics 21, 67 (1978).
[17] V. A. Vavilin, A. N. Zhabotinsky, and A. N. Zaikin, A Study of a Self-Oscillatory Chemical Reaction I: The Autonomous System. In: Biological and Biochemical Oscillators (B. Chance, E. K. Pye, A. K. Ghosh, and B. Hess, eds.), pp. 71-79. Academic Press, New York 1973.

[18] R. A. Schmitz, K. R. Graziani, and J. L. Hudson, J. Chem. Phys. 67, 3040 (1977).

[19] K. R. Graziani, J. R. Hudson, and R. A. Schmitz, The Chem. Engng. J. 12, 9 (1976).

[20] O. E. Rössler, Chaos in Realistic Reaction Systems, Lecture held at the ,Chemiedozententagung“", Marburg, March 1977.

[21] O. E. Rössler and K. Wegmann, Nature London 290, $89-90$ (1978).

[22] O. E. Rössler and K. Wegmann, Different Types of Chaos in the Belousov-Zhabotinsky Reaction. Lecture held at the 13th Symposium on Theoretical Chemistry, Münster, October 1977.

[23] The value of $5.6 \mathrm{M} \mathrm{H}_{2} \mathrm{SO}_{4}$ described in [21] is a misprint and should read " 1.4 ".

[24] R. FitzHugh, Biophys. J. 1, 445 (1961).

[25] A. T. Winfree, Science, Wash. 175, 634 (1972).

[26] An observation first reported by J.C. Roux (personal communication 1977).

[27] L. F. Olsen and H. Degn, Nature London 267, 177 to 178 (1977).

[28] One straightforward reduced model consists of the 2 -variable $\mathrm{HBrO}_{2} / \mathrm{Br}^{-}$model of ref. [7], adding $\mathrm{Ce}^{3+}$ or $\mathrm{Mn}^{2+}$ (in the role of a constant influx exhaustible precursor to the autocatalyst) as a third variable.

[29] Added in proof: Two coexisting limit cycles were also observed by the Bordeaux group (C. Vidal, personal communication 1978). 